\title{
Spatial pattern learning in rats: Conditional control by two patterns
}

\author{
MICHAEL F. BROWN, ELIZABETH DIGELLO, MICHELLE MILEWSKI, \\ MEREDITH WILSON, and MICHAEL KOZAK \\ Villanova University, Villanova, Pennsylvania
}

\begin{abstract}
Rats searched for food hidden on top of poles in a $4 \times 4$ matrix of poles. Before each trial, the location of the four baited poles was unpredictable. However, the poles were always baited in one of two spatial patterns, either a square or a line. The food hidden on the four baited poles was one of two types, and the food type determined the identity of the pattern. Thus, once one baited pole was discovered, the food type provided a cue for the spatial pattern in which the remaining food was hidden. The results showed that rats learned the two spatial patterns in which the food was hidden and used the conditional cue to determine which pattern was relevant on individual trials, thereby increasing the efficiency of spatial search.
\end{abstract}

Many studies and theoretical analyses of spatial learning in animals have focused on the means by which animals use spatial cues (landmarks) to discriminate their own spatial location or the locations of goals (e.g., Cartwright \& Collette, 1983; Cheng, 1989; Cheng \& Spetch, 1999; Gallistel, 1990; Greene \& Cook, 1997; Morris, 1981; Olton, 1978; Poucet, 1993; Thinus-Blanc, 1996). There is also clear evidence that animals can use internal cues to track their movement through space ("dead reckoning" or "path integration"; e.g., Brown \& Moore, 1997; Etienne, Maurer, \& Saucy, 1988; Gallistel, 1990; McNaughton, Chen, \& Markus, 1991). Cheng (1986; Cheng \& Gallistel, 1984) has argued that the geometric properties of the environment can be used as a spatial cue.

Brown and Terrinoni (1996) recently provided evidence for a different type of spatial cue. They showed that rats locate hidden goals using the spatial pattern of the goals. Specifically, food pellets were hidden on top of vertical poles. Rats determined whether a pole was baited by rearing up on their hind limbs and examining the well on the top of the pole. At the beginning of each trial, the location of the baited poles was unpredictable. However, the poles always formed a particular spatial pattern. In one experiment, the four baited poles always formed a $2 \times 2$ square within the $5 \times 5$ matrix of poles. In a second experiment, the four or five baited poles formed a line within the $4 \times 5$ matrix of poles. Thus, the location of one or more baited poles in combination with knowledge of the pattern in which poles were baited provides information about the location of the remaining baited poles. Brown and Terrinoni's measures and analyses led them to conclude that rats were able to use the consistent spatial pat-

Additional information about this research, including a video clip of a typical trial of the procedure, is available on the Internet at www. homepage.villanova.edu/michael.brown. Correspondence should be addressed to M. F. Brown, Department of Psychology, Villanova University, Villanova, PA 19085 (e-mail: michael.brown@villanova.edu). tern of baited poles in order to narrow their search of the poles, thereby more efficiently locating the food. This result has been replicated by Lebowitz and Brown (1999), who found a higher level of control by a spatial pattern in male rats than in female rats.

What distinguishes the processes involved in Brown and Terrinoni's (1996) task from previous studies of spatial cognition is that the spatial relations controlling behavior are relations among locations that are not distinguished by any perceivable cue. A number of studies have indicated that spatial patterns or geometric relations among perceived spatial locations control behavior (Cheng, 1986; Greene \& Cook, 1997; Kamil \& Jones, 1997; Spetch et al., 1997). However, the spatial pattern that controlled the choices of Brown and Terrinoni's rats was a pattern among hidden food items. Thus, this form of pattern learning appears to require an abstract representation of spatial relations that is not anchored to visual or other perceived cues.

In the present set of experiments, we examined the possibility that two spatial patterns (a square and a line) can simultaneously control spatial choice. The pattern according to which poles were baited on each trial was determined by the identity of the food used as bait on that trial. For half the rats, four poles forming a square were baited when the bait was a sucrose pellet, but four poles forming a line were baited when the bait was a sunflower seed half. The other half of the rats had the reverse assignment of patterns and baits. Thus, the pattern according to which poles were baited could be determined only after the food type had been identified (i.e., when the rat found the first baited pole). The analyses followed those used by Brown and Terrinoni and were designed to determine whether rats' choices were controlled by the spatial pattern, for which the identity of food served as a conditional cue. It should be pointed out that these analyses involve only a restricted set of the rats' choice behavior, which allows control of behavior by the spatial pattern to be isolated from other factors that affect choices. 
Because of the restrictions, only a portion of the experimental trials provide the data included in the primary analysis.

The first experiment was conducted in two replications, differing in the number subjects and the number of experimental trials conducted.

\section{EXPERIMENT 1}

\section{Method}

Subjects. The subjects were 10 (Experiment $1 \mathrm{~A}, \mathrm{E} 1 \mathrm{~A}$ ) and 20 (Experiment 1B, E1B) male Sprague-Dawley rats, approximately 4 months of age when the experiment began. Starting 1 week prior to the beginning of the training protocol described below, the rats were maintained on a diet of approximately $15 \mathrm{~g}$ of Rat Chow per day, provided immediately following any experimental testing. The subjects were housed in groups of 2 or 3 , in a standard colony with a 12:12-h light:dark cycle. All experimental procedures occurred during the dark phase of the cycle.

Apparatus. The apparatus was a $120 \times 58 \times 33 \mathrm{~cm}$ tall box, constructed of $1.5-\mathrm{cm}$-thick plywood and painted flat black. The top of the box was open. Centered in the box was a $4 \times 4$ matrix of $15.5-\mathrm{cm}$-tall poles, separated by $13 \mathrm{~cm}$. Each pole was $1.2 \mathrm{~cm}$ in diameter and had a 0.5 -cm-deep well drilled into its top end. The size of this well resulted in visual occlusion of the food items used to bait the poles but also allowed the rats to extract the food from the wells with their tongues. A 2-cm-deep layer of wood shavings covered the floor of the apparatus. The apparatus was in a laboratory room illuminated by fluorescent tubes.

A set of four additional poles was also used during the training phase of the experiments. These four poles were separated by $7 \mathrm{~cm}$ and differed in height $(2.0,4.5,12.5$, and $15.5 \mathrm{~cm})$. They were mounted in a line in order of height on a piece of thin plywood, so that they could be easily removed from the apparatus.

Training procedure. The rats were first given 10-20 of each of the two food items to be used in the experiment, during each of the 3 days prior to placement in the apparatus. These two food items were 45-mg sucrose pellets (BioServe, Inc.) and halves of shelled sunflower seeds. The rats were then trained to obtain food items from the poles. The set of four poles described above was placed in one of the open ends of the apparatus, with the $4 \times 4$ matrix of poles blocked by a section of black poster board. On each training trial, each of the four poles was baited with one sucrose pellet or one sunflower seed half. In addition, during the first two or three trials, food items were scattered on the floor of the apparatus immediately surrounding the poles. Two trials occurred per day for 6 ( $E / A)$ or 7 (EIB) days, with each of the two food types used during one trial per day (randomly ordered). A trial ended when the rat had eaten all of the food items or $5 \mathrm{~min}$ had elapsed. Two rats in E1B that did not consistently consume the pellets from the training apparatus by the end of the training procedure were eliminated from the experiment.

Testing procedure. The set of four poles used during training was not present during the experimental trials. The rats were tested for a total of 30 (E1A) or 40 (E1B) trials, with two trials occurring each day. During one of the two daily trials, four poles were baited forming a $2 \times 2$ square. During the remaining daily trial, four poles were baited forming a line. The order in which these two trial types occurred each day was randomized. For 5 (randomly chosen) rats in E1A and 9 rats in E1B, the poles were always baited with sucrose pellets when the baiting pattern was a square and with sunflower seeds when the pattern was a line. For the remaining 5 rats in E1A and 9 rats in $\mathrm{ElB}$, these assignments were reversed. Given the $4 \times 4$ matrix of poles, there were nine specific square configurations of
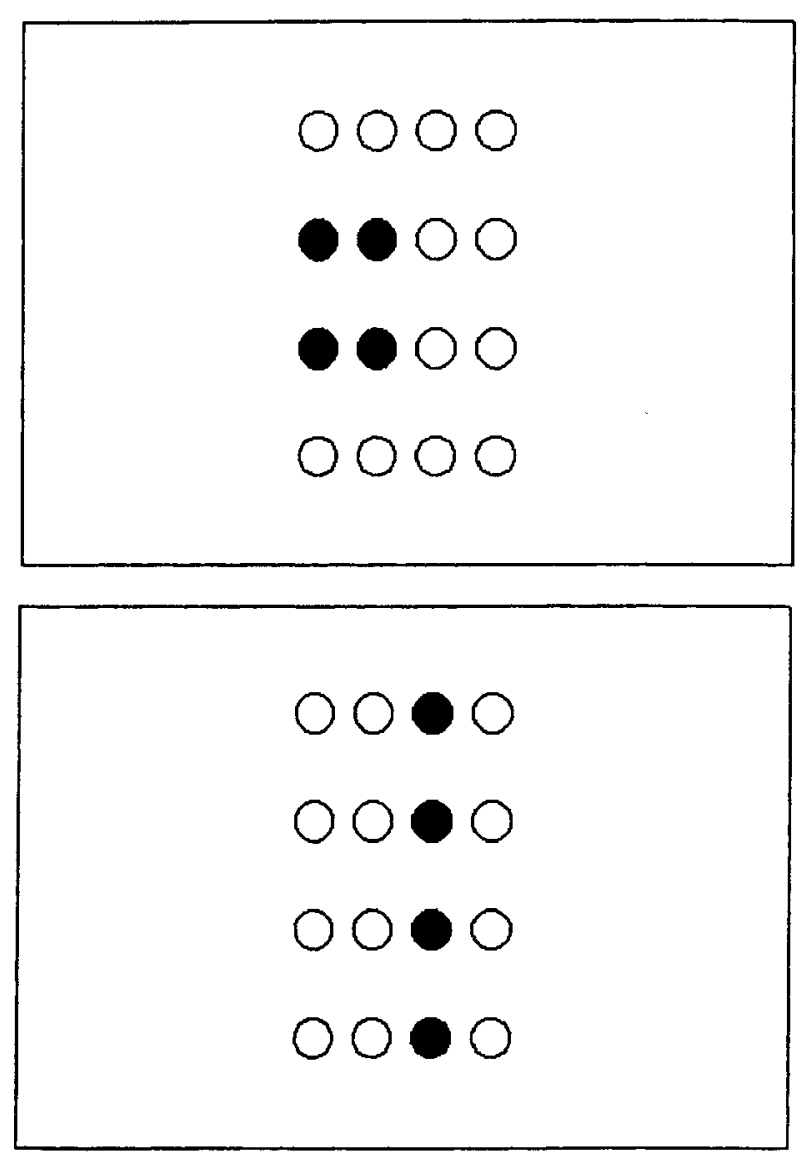

Figure 1. Schematic overhead view of the apparatus, showing an example of the configuration of baited poles during square (top panel) and line (bottom panel) trials. Before each trial, the specific location of the pattern was unpredictable.

baited poles and eight specific line configurations. Figure 1 shows one example of each. For each trial, the specific set of poles baited was chosen randomly from these eight (or nine) possibilities.

At the beginning of each trial, the rat was placed in the open area at one end of the apparatus. It was then allowed to choose poles until all four baited poles had been chosen or until $5 \mathrm{~min}$ had elapsed. A choice was defined when the rat touched the top of a pole with its tongue or snout. The experimenter recorded the sequence of poles chosen by the rat.

\section{Results}

Experiment 1A. All 10 rats quickly obtained all the pellets by the end of the 12 training trials. As a result of experimenter error, poles were baited with the wrong food type on Test Trial 25 for 3 subjects. Thus, the data from the last 6 trials for these subjects were not included in the analyses reported below.

During the 30 (or 24) trials of testing, the rats chose a mean of 10.6 different poles before finding all four baited poles. (Note that revisits to previously visited poles are not included in these analyses.) On the basis of a Monte Carlo simulation, it would be expected that they would 
choose a mean of 13.7 different poles before locating the four baited ones, if choices were made randomly. These two values differ reliably $[t(9)=11.1]$.

One trivial explanation for the efficiency of the rats' choices is that they could perceive the presence of the food (e.g., by smell). If so, it would be expected that they could locate the first baited pole more efficiently than expected by chance. However, they chose a mean of 3.22 different poles before locating the first baited pole, which did not differ from the value expected by chance (3.45) $[t(9)=1.2]$. Thus, it appears that discovery of one baited pole was required for the rats to locate additional baited poles at levels above that expected by chance.

Search efficiency could be increased simply by searching in the general vicinity of the first baited pole. Thus, in order to determine whether choices were controlled by the pattern in which baited poles were arranged, a more sophisticated measure is needed. The primary measure we used is the one developed by Brown and Terrinoni (1996). It looks at a restricted set of pole choices and determines whether choices of poles conformed to the pattern to a greater extent than would be expected on the basis of chance. The choices examined in this analysis were restricted in several ways. First, only the choice immediately following discovery of the second baited pole and the choice immediately following the third baited pole were examined. As illustrated in Figure 2, these choices have special properties, which reveal control of spatial choice by the spatial pattern in which poles are baited. Second, only choices of poles that were spatially adjacent to the just-discovered baited pole were examined. This restriction was imposed for two reasons: (1) to control for any effects of spatial proximity on choice, and (2) because most $(64.6 \%)$ choices were to poles adjacent to the previously chosen pole. Spatially adjacent poles are those which are adjacent in either a row or column. Finally, poles that had been previously visited were not included in the analyses, in order to control for any effect of previous visits to poles on the tendency to subsequently choose that pole (e.g., rats might avoid revisits to poles as they avoid revisits to arms in the radial maze; e.g., Olton \& Samuelson, 1976).

As for the experiments of Brown and Terrinoni (1996), the number of moves to previously unvisited, adjacent poles following discovery of the second baited pole and following discovery of the third baited poles was determined. For each rat, the proportions of these moves that conformed to the pattern (square or line) were determined. These proportions served as the primary empirical measure of performance. These proportions were compared with an estimate of the proportion expected by chance. This estimate of chance was calculated by first determining the number of previously unvisited, adjacent poles available at the time the included choices were made. For each rat, the proportion of the available poles that conformed to the baiting pattern was determined. As an illustration, consider the trial represented in the top panel of Figure 2. If the pattern is a square, then the es- timate of chance is 2 (poles that conform to the square pattern) $/ 3$ (total poles that meet the restrictions) $=.67$. It should be noted that the actual estimates used were calculated over blocks of trials for individual rats and that these estimates are determined by a number of factors including whether or not the pattern on a particular trial is on the edge of the matrix, the number of adjacent poles that have already been visited, and so on (for details, see Brown \& Terrinoni, 1996). Given that a choice was made that met our restrictions (i.e., from the justdiscovered second or third baited pole to an adjacent, previously unvisited pole), control by the spatial pattern would be indicated if the obtained proportion of moves to poles conforming to the pattern was greater than this estimate.

Figure 3 shows the mean proportion of moves to adjacent, previously unvisited poles obtained and expected. An analysis of variance (ANOVA) revealed that the overall difference between obtained and expected values was reliable $[F(1,9)=26.0]$. The difference between obtained and expected values was greater following discovery of the third baited pole than following discovery of the second baited pole (as revealed by the discovery number $x$ expected vs. obtained interaction term) $[F(1,9)=9.6]$. There was no evidence that the magnitude of the difference between obtained and expected proportions varied for the two spatial patterns (pattern $\times$ expected vs. obtained interaction term) $[F(1,9)<1]$, nor was there any evidence for a three-way interaction among these factors $[F(1,9)=1.5]$.

The number of poles chosen prior to discovery of the first baited pole provides no evidence that rats can perceptually detect the presence of the food items. However, it remains possible that, once a rat is choosing poles in the general vicinity of the baited poles, it becomes possible to see or smell the food in nearby poles. Thus, an additional analysis was performed to look for evidence of such an effect (see Brown \& Terrinoni, 1996, p. 443). This analysis rests on the fact that, following discovery of the second baited pole when poles are arranged in a square, some poles conform to the square pattern but are not actually baited (refer to the top panel of Figure 2). If choices under these conditions are controlled by the square pattern, then rats should not choose the actually baited poles over the unbaited (but possibly baited according to the pattern) pole any more than expected by chance. On the other hand, if rats' choice efficiency is produced by an ability to smell or otherwise perceive the food, then they should have a tendency to choose the actually baited pole over one that conforms to the pattern but is not actually baited. The proportion of moves to poles conforming to the square pattern that were to actually baited poles was .91 . The proportion of available poles conforming to the pattern that were actually baited when these moves occurred was .88 . These two proportions do not differ $[t(9)<1]$.

Experiment 1B. Our plan was to examine the obtained and expected proportions of critical choices that 

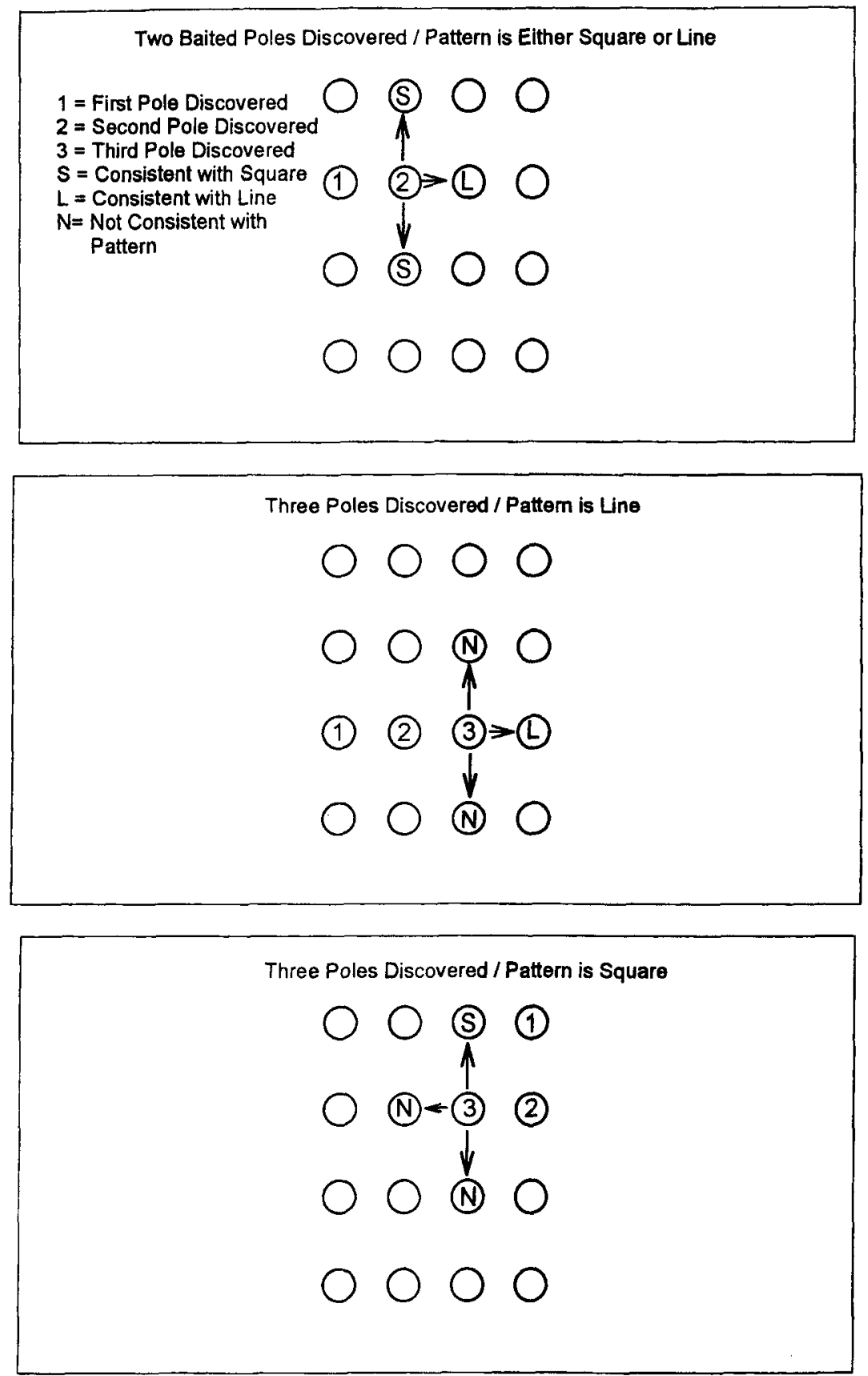

Figure 2. Illustration of the logic of the primary data analysis. Following the discovery of two adjacent baited poles (top panel), up to two adjacent poles are consistent with the square pattern and up to one adjacent pole is consistent with the line pattern. Following discovery of three adjacent baited poles, the location of the last baited pole is determined by the square (middle panel) or the line (bottom panel) pattern.

conformed to the spatial pattern over the course of four blocks of 10 trials each. Unfortunately, there was an insufficient number of choices following discovery of the third baited pole to allow this analysis ( 5 rats provided no cases of such choices in at least one trial block). Therefore, Figure 4 shows the obtained and expected proportions of choices conforming to the pattern following dis- covery of the second baited pole over the course of four trial blocks and the corresponding proportions following discovery of the third baited pole over the course of two trial blocks. To allow comparison of performance following the second and third discoveries, a 2 (proportion) $\times 2$ (discovery) $\times 2$ (blocks of 20 trials) ANOVA served as the primary inferential analysis. This analysis revealed 

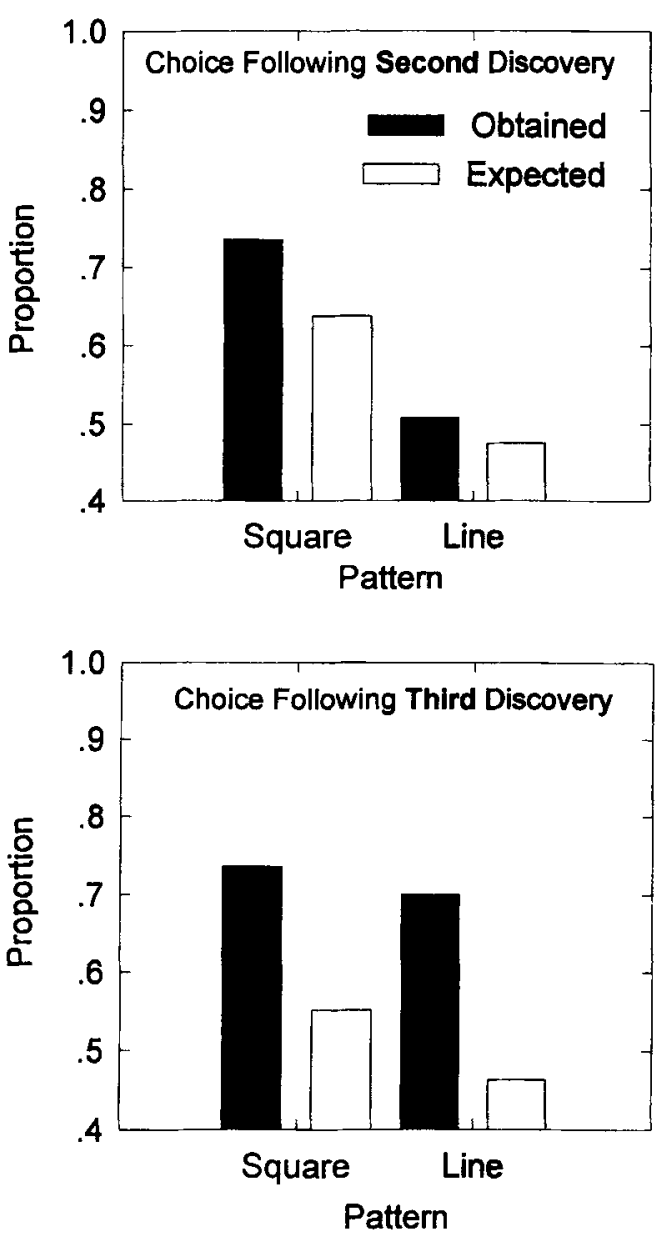

Figure 3. The primary results of Experiment 1A. The proportion of choices (within the restrictions described in the text) following the discovery of the second (top panel) and third (bottom panel) baited pole directed to a pole that is consistent with the baited pattern. The proportion obtained and the proportion that would be expected on the basis of chance are shown for both the square and the line pattern.

that the obtained proportion of choices conforming to the pattern was greater than the expected proportion $[F(1,17)$ $=11.4]$. However, there was no evidence that the magnitude of this difference varied with either block or discovery, as indicated by the lack of proportion $\times$ block $[F(1,17)<1]$ and proportion $\times$ discovery $[F(1,17)=1.6]$ interaction terms. Nor was there a three-way interaction among the effects of these variables $[F(1,17)<1]$.

Because it was possible to obtain finer resolution of changes in performance over the course of the experiment for choices following discovery of the second baited pole, a second ANOVA was conducted on those data, in which performance was segmented into four blocks of 10 trials each (see Figure 4). This 2 (proportion) $\times 4$ (block) ANOVA provided only ambiguous evidence for a difference between obtained and expected proportion $[F(1,17)=$ $3.9, p=.06]$. There was no evidence for a change in the relation between obtained and expected proportions, as indicated by the lack of a proportion $\times$ block interaction term $[F(3,51)=1.3]$.

As in Experiment $1 \mathrm{~A}$, an analysis was done to examine the possibility that there was a tendency to choose poles that were actually baited in comparison with poles that conformed to the baited pattern but happened not to be baited. It should be noted that such poles exist only following the second discovery and only in the case of the square pattern (see Figure 2). If this tendency exists, it would suggest that rats' choices are determined by the presence of food on the poles: For example, they might be able to see or smell the food. Among choices of poles conforming to the square pattern following discovery of the second baited pole, a mean (over rats) of .77 of them were to poles that actually contained food. This value was compared with the mean (over rats) proportion of poles conforming to the pattern that were actually baited (.74). These two values were not reliably different $[t(17)<1]$.
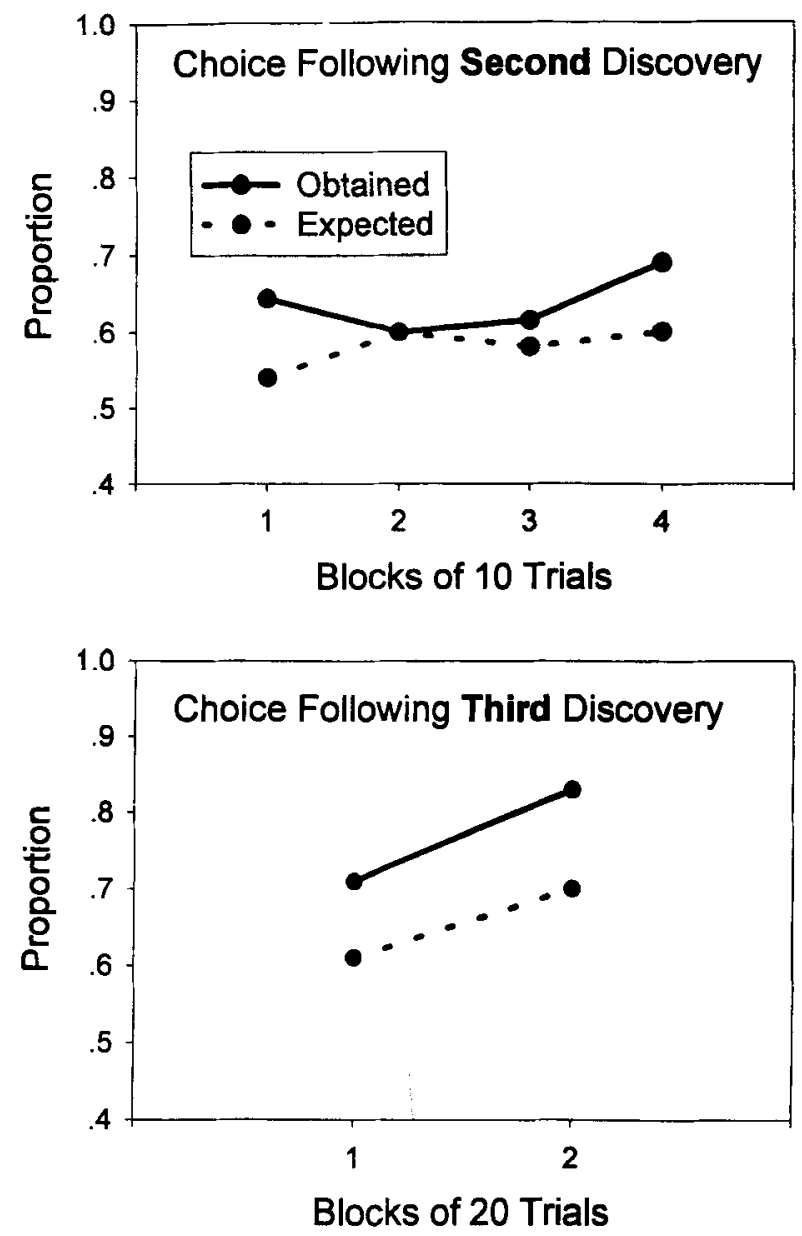

Figure 4. The primary results of Experiment 1B. The proportion of choices (within the restrictions described in the text) following the discovery of the second (top panel) and third (bottom panel) baited pole directed to a pole that is consistent with the baited pattern. The proportion obtained and the proportion that would be expected on the basis of chance are shown as a function of trial blocks. The results are collapsed for the two spatial patterns. 


\section{Discussion}

These data provide evidence that spatial choice in rats can be controlled by two different patterns, with the pattern controlling behavior on a particular trial determined by the identity of a conditional cue. This basic result was obtained in each of these two experimental replications.

That two directly conflicting spatial patterns come to conditionally control choices within the 30 or 40 trials of these experiments is surprising. Brown and Terrinoni (1996) found that either a square pattern or a linear pattern reliably affects choices within 30 experimental trials. However, one might predict that conditional control by two different patterns would be substantially more difficult to acquire. Certainly, one would expect a learning period in this experimental task, in the sense that the relation between the cue and the pattern in which poles are baited can be determined only by exposure to that relation. With the small number of subjects and the small number of trials used in Experiment $1 \mathrm{~A}$, it was not possible to resolve performance into trial blocks in order to examine the nature of the learning curve. Experiment $1 \mathrm{~B}$ did allow performance to be resolved in trial blocks to some extent to allow acquisition of control by the patterns to be examined. However, the data provide no evidence that control of choices by the spatial patterns increased over the course of the experiment. This finding is somewhat troublesome because it seems clear that conditional control by the two spatial patterns requires that both the pattern and the relationship between the food types and the patterns be learned. It may, of course, be that this learning occurs within the 10 or 20 trials that formed trial blocks in Experiment 1B. Before concluding that conditional control by square and linear patterns of baited poles is acquired within 10 or 20 trials, however, we felt it important to more completely rule out two alternate possibilities.

\section{EXPERIMENT 2}

Two lines of evidence in the experiments reported above argue against the possibility that odor cues affected pole choices. First, there was no evidence that the number of choices required to locate the first baited pole was less than chance in either experiment. Second, there was no evidence that the rats were more likely to choose baited poles that conformed to the pattern than to choose unbaited poles that conformed to the pattern. However, both of these are null results, and it is possible that a subtle influence of odor cues was not detected by these measures but did affect the primary performance measures. We used a technique in Experiment 2 that rules out any influence of odor. In particular, both a pellet and a half sunflower seed were embedded under a nylon mesh screen in the well in each pole. Poles were baited by placing a pellet or seed on top of this mesh. Thus, any odor that might be detectable from a baited pole was present in all poles.
A second concern is the possibility of observer bias in the measurement of pole choices. Choices were coded by the experimenter, using the criterion of the rat touching the top of a pole. Although this operational definition of a choice seems to leave no room for a role of observer bias, and there appeared to be few, if any, ambiguous examples of choice-related behavior, it remains possible that experimenters had a more lax criterion for choices of poles that contained a pellet or conformed to the pattern than for poles that did not. (It should be noted that the lack of evidence for a greater tendency to choose baited poles that conformed to the pattern compared with unbaited poles that conformed to the pattern suggests that such a hypothetical bias would be based on conformity to the pattern rather than the presence of bait.) In Experiment 2 , we attempted to avoid any possibility of measurement bias by developing an automated version of the pole box. Unfortunately, this attempt failed: Our automated pole box did not measure choices reliably. However, because all trials of the experiment were videotaped, we were able to examine the data for evidence of observer bias.

\section{Method}

Subjects. Fifteen male Sprague-Dawley rats served as subjects. They were approximately 3.5 months old at the start of the experiment and were maintained in the same manner as the subjects in Experiments $\mathrm{IA}$ and $\mathrm{IB}$.

Apparatus. The apparatus was the same as that used in Experiments $1 \mathrm{~A}$ and $\mathrm{BB}$, modified so that choices could be measured electronically and so that the odor of bait was present in all poles. A length of PVC tube (inside diameter, $1.5 \mathrm{~cm}$; outside diameter, $2.0 \mathrm{~cm}$ ) surrounded each pole, extending $1.5 \mathrm{~cm}$ above the top end of the pole. The bottom end of the PVC tube was attached to a needle, which extended through a hole in the floor of the apparatus and rested on a microswitch. To investigate the contents of a pole (thereby making a choice), the rat pressed down on the PVC tube, thereby closing the microswitch and allowing access to the food well (and food, if the choice was a correct one). The spring on the microswitch returned the PVC tube to its proper height following a choice. The microswitches were associated with digital input/output circuit cards in a computer, which detected choices and signaled the experimenter when the trial was completed. To allow free vertical movement of the PVC tubes, wood shavings were not placed on the apparatus floor in this experiment. The sucrose pellets used in this experiment (Noyes, Inc., Formula F, $45 \mathrm{mg}$ ) were different from those used in Experiments $1 \mathrm{~A}$ and $\mathrm{IB}$.

Starting with the 13th test trial (see Results section below), the apparatus was modified such that pellets and sunflower seed halves were present in the well of each pole. Each well was drilled to a depth of $1.0 \mathrm{~cm}$. One sucrose pellet and one sunflower seed half were placed in each well. A small piece of nylon mesh was placed on top of these food items, forming a surface on which additional food could be placed. A metal washer was then glued on top of the pole, forming a rim that held the nylon mesh in place and visually occluded any bait. Poles were thereafter baited by placing a sucrose pellet or seed half on top of the nylon mesh. Thus, any odor given off by bait was present in all poles, but only poles with a pellet or seed on top of the mesh had accessible food items.

Two sets of training poles were used in this experiment. Each set consisted of four poles that differed in height $(2.0,4.5,12.5$, and $15.5 \mathrm{~cm}$ ). PVC tubes the same height as each pole surrounded each 
training pole. Both sets were mounted on cardboard surfaces, so that they could be easily placed in and removed from the apparatus. In both sets, adjacent poles were separated by $7 \mathrm{~cm}$. One set was spatially arranged in a line, and the other was spatially arranged in a square.

Training procedure. Each rat received approximately $10 \mathrm{su}-$ crose pellets and 10 sunflower seed halves in its home cage during each of the 3 days prior to the beginning of training. Seven rats were randomly assigned to receive sucrose pellets in the square pattern and sunflower seed halves in the line pattern; for the remaining rats, these assignments were reversed. During each training trial, a Plexiglas barrier was placed in the pole matrix so that the rat had access to half of the pole matrix and the open area in one end of the apparatus. A training apparatus was placed in the open area. The food used to bait the poles alternated from trial to trial. The training apparatus appropriate for the food type being used was baited and placed in the apparatus, as was a randomly chosen square (or line) in the pole matrix. In addition, during the first two training trials, approximately 12 pellets (or seed halves) were scattered on the floor of the apparatus. Training trials ended when the rat had chosen the four baited poles in the matrix or $6 \mathrm{~min}$ had elapsed. Training was considered complete for an individual rat when it met the former criterion on two consecutive trials.

Testing procedure. The pattern (square or line) was chosen for each subject in randomized blocks of 10 trials ( 5 trials with each pattern per block). The location of the pattern within the $4 \times 4$ pole matrix was randomly chosen from among the nine squares or eight lines available. The rat was placed in one end of the box and was allowed to make choices until all four baited poles had been chosen or $6 \mathrm{~min}$ had passed. Choices were measured both by the electronic system and by the experimenter. All trials were videotaped by a camera, which was mounted above the apparatus.

Each rat was tested for 52 trials. One trial per day was conducted for 31 days, followed by two trials per day for 11 days.

\section{Results}

Two possibly related problems quickly became apparent. First, the electronic choice detection system was not reliable because the rats were able to sometimes remove food from baited poles without closing the microswitch. Thus, choices were clearly going undetected by the electronic system. Second, it appeared to us that the rats might be able to detect the absence of food by placing their snout above the PVC tube, but without touching it This is very much unlike the choice behavior we have observed previously in the pole box, in that rats have always touched the top of the pole after approaching within 1 or $2 \mathrm{~cm}$ of it. One possibility that occurred to us is that the PVC tube may concentrate odor molecules from the bait in the area above the pole, allowing odor to become a cue in this modified apparatus. For these reasons, we implemented two changes starting with Trial 13. First, all choices were coded by the experimenter, with a choice defined by the rat's snout touching or being directly above the pole. Second, the odor of the two food types was added to all 16 poles, as described in the Method section. All results described below are from the $\mathbf{4 0}$ trials that followed these changes.

Trials were analyzed in two blocks of 20 trials each, with the exception of 1 rat that had only 19 trials in Block 2 due to experimenter error. During Blocks 1 and 2, the rats chose a mean of 3.7 and 3.6 different poles before
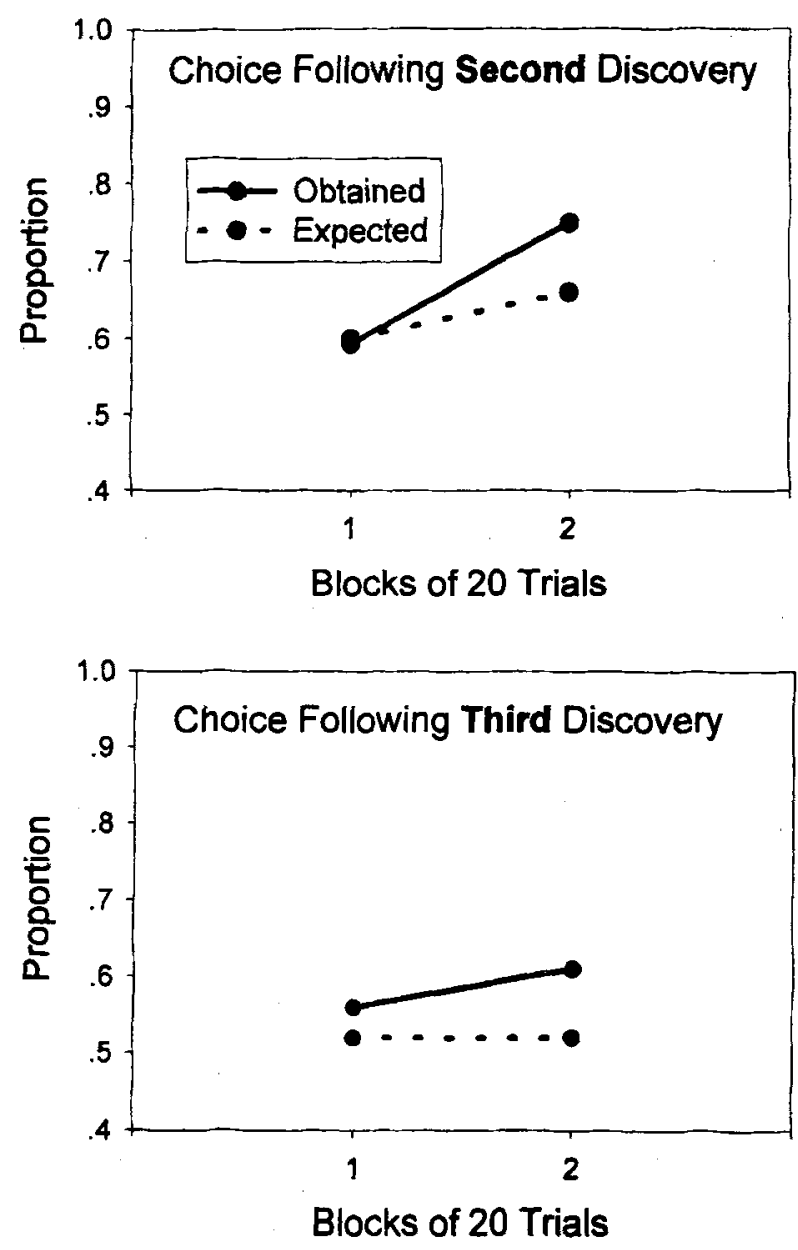

Figure 5. The primary results of Experiment 2. The proportion of choices (within the restrictions described in the text) following the discovery of the second (top panel) and third (bottom panel) baited pole directed to a pole that is consistent with the baited pattern. The proportion obtained and the proportion that would be expected on the basis of chance are shown as a function of trial blocks. The results are collapsed for the two spatial patterns.

locating a baited pole, respectively. Thus, the rats did not locate the first baited pole more efficiently than would be expected by chance ( 3.45 poles). During Blocks $l$ and 2 , the rats chose a mean of 12.8 and 12.5 different poles before locating all four baited poles, respectively. Although the two values do not differ reliably $[t(14)<1]$, both values are reliably less than the number expected by chance (13.7) $[t \mathrm{~s}(14)=4.2$ and 5.0, respectively].

The results of the analysis of choices following discovery of the second and third baited poles are summarized in Figure 5 . These data were analyzed using a 2 (discovery) $\times 2$ (block) $\times 2$ (pattern: square vs. line) $\times 2$ (obtained vs. expected) ANOVA. Only those factors that include the last variable are theoretically meaningful, and the results are collapsed across pattern in Figure 5, because no factors including that variable produced significant effects. The ANOVA revealed a significant interaction 
between block and obtained versus expected $[F(1,11)=$ 5.92]. The form of the interaction was that there was no difference between the expected and obtained proportions during Block $1[F(1,13)<1]$, but the obtained proportion was reliably greater than the expected proportion during Block $2[F(1,13)=6.6]$.

As in Experiments $1 \mathrm{~A}$ and $1 \mathrm{~B}$, we more closely examined the choices made following discovery of the second baited pole when the pattern was a square. The analysis was to determine whether choices to baited poles conforming to the pattern in comparison with unbaited poles that conformed with the pattern were more likely than expected on the basis of chance. Among choices following discovery of the second baited pole and conforming to the baited square pattern, .82 of them were to a pole that was actually baited. Of the poles available at the time of those choices, .78 of them were actually baited. The two proportions are not significantly different $[t(14)=1.4]$.

We were not able to use the automated apparatus to examine the possibility of observer bias. As an alternative, we used the videotape records of trials to seek evidence for an effect of observer bias. Videotape of the last 10 trials was coded by the same experimenter who had observed and coded the behavior originally. However, the contrast on the video monitor was adjusted such that it was impossible to detect the bait on the poles in the video. Thus, the coder had no knowledge of the location of the baited poles in the videotaped trials. Choice sequences as coded originally were compared with choice sequences coded from the videotape. Of choices originally coded, $99.1 \%$ were also coded from the videotape. A direct search for observer bias involves comparing the number of disagreements between the original and videotape codings that favor pattern learning (choices consistent with the pattern that appear only in the original coding or choices inconsistent with the pattern that appear only in the videotape coding) with disagreements that do not favor pattern learning (choices inconsistent with the pattern that appear only in the original coding or choices consistent with the pattern that appear only in the videotape coding). There were five disagreements consistent with a bias to see choices consistent with the pattern and four disagreements inconsistent with such a bias. Thus, there is no evidence that observer bias played a role in the evidence for control by the spatial pattern.

\section{Discussion}

The techniques used in Experiment 2 enhance the evidence for conditional control by spatial patterns in two ways. First, although our attempt to automate the pole box did not succeed, our analysis of choice sequences coded without knowledge of the location of baited poles rules out the possibility that our measure of choice is biased to favor choices that are consistent with the spatial pattern. Second, although Experiments $1 \mathrm{~A}$ and 1B pro- vided evidence against the possibility that perceptual cues provided information about the location of baited poles, the use of sham bait in Experiment 2 even more strongly rules out odor cues as being involved in choice accuracy in the pole box.

In addition, the results of Experiment 2 include increasing control by the spatial patterns with experience. Such an acquisition curve is to be expected, given that the rats must learn both the patterns themselves and the relationship between the conditional cue (bait type) and pattern. It is not clear why acquisition of control by the pattern was detected in Experiment 2 but not in Experiment 1B. In both experiments, the ability to make spatial choices consistent with either of two spatial patterns and depending on the bait type occurred rapidly, developing within 10 or 20 trials.

\section{GENERAL DISCUSSION}

These three experiments all show the same basic set of behavioral phenomena. Like the earlier experiments of Brown and Terrinoni (1996), they demonstrate control of choices by the spatial pattern in which hidden food is located. In this case, however, individual rats were controlled by two spatial patterns, and a conditional cue determined which one controlled choices on a particular trial. Conditional control by two spatial patterns was acquired rapidly, with the effect being detectable within 10 or 20 trials. On the other hand, the terminal level of performance shown by the rats in these experiments appears to be lower than those shown when food is baited in only one pattern (Brown \& Terrinoni, 1996).

It should be emphasized that the behavioral indicators of control by the square pattern and control by the line pattern are not just different but are in fact members of complimentary sets. We measured the proportion of choices to adjacent (unbaited) poles that conformed to the pattern. Following discovery of the second baited poles, all such moves conform either to a square or a line pattern (see the top panel of Figure 2). This point is important because it addresses a potential alternative to conditional control as an explanation of the basic result. Specifically, one might propose that some rats learned one pattern and other rats learned the other pattern, resulting in the overall pattern of results we obtained. However, if an individual rat was controlled by only one pattern, then its choice behavior would be in contradiction of the other pattern. Thus, one would expect a negative correlation between choice accuracy with one pattern and choice accuracy with the other. As a direct test of this prediction, we compared the difference between expected and obtained proportions of choices conforming to each pattern after the discovery of the 2 nd baited pole for each of the 43 rats completing the three experiments. The correlation between the difference score for the square pattern and the difference score for the line pattern was not neg- 
ative $(r=0.253, p=.10)$. Thus, there was conditional control of choice behavior, as determined by the cued pattern, within individual subjects.

The existence of conditional control by two spatial patterns indicates a high level of flexibility in the use of spatial representations. The spatial pattern appears to be a coherent representational unit, which can be associated with a conditional cue. This level of flexibility would serve animals well in nature, where multiple spatial patterns of various resources are undoubtedly common.

In addition, the demonstration of conditional control by multiple patterns provides important constraints on our understanding of the mechanism of spatial pattern learning. Brown and Terrinoni (1996) described an alternative to pattern learning as an explanation for control by a square pattern. The alternative involves spatial generalization from the location of baited poles that have been discovered to adjacent poles. Following the discovery of two adjacent baited poles, the overlap of the corresponding generalization gradients could be expected to produce more generalized excitation to the poles that are consistent with the square pattern than to the poles that are not (see Brown \& Terrinoni, 1996, pp. 443-444, for more details). Simultaneous control by a square pattern (which can be explained by this alternative) and a line pattern (which is inconsistent with this explanation) provides a strong refutation of this alternative explanation.

Control by spatial patterns appears to require at least two sets of processes. First, the nature of the pattern must be learned. There are at least two general ways in which this might occur. First, rats might form a representation of the geometric properties of the pattern, akin to the representations of the geometric properties of visible environments that have been described (Cheng, 1986; Cheng \& Gallistel, 1984). Second, control by a spatial pattern might come in the form of a response tendency, such that rats tend to turn in a perpendicular direction after finding two baited poles in a row (square pattern) or tend to continue moving in the same direction after finding two baited poles in a row (line pattern). Recent data from our laboratory favor the former explanation (Zeiler \& Brown, 2000).

The second set of processes required for control by a spatial pattern of hidden food is a means of keeping track of the location of previously chosen poles so that their location, in combination with the nature of the pattern, can provide information about the location of baited poles that remain. For example, a rat that has come under the control of a square pattern must keep track of the locations of two discovered baited poles in order to determine where the other two corners of the square are. We have not yet begun a systematic examination of this aspect of the pole box task. Although no visual landmarks are available in the pole box itself, extra-apparatus cues could be used to discriminate among individual poles. The visual properties of a pole as an element in the vi- sual texture of the entire matrix of poles might also provide information that could be used to distinguish individual poles. Alternatively, rats could use internal cues (vestibular or proprioceptive information) to dead reckon (Etienne, Maurer, \& Séguinot, 1996; Gallistel, 1990) their current location relative to previously discovered baited poles.

The behavior of rats can be controlled by serial patterns of reinforcement magnitude (Fountain \& Hulse, 1981; Hulse \& Dorsky, 1977) and by serial patterns of change in the temporal properties of reinforcement (Church \& Lacourse, 1998). Control of spatial choices by the pattern of hidden food items in the pole box is a demonstration of control by a pattern in the medium of space. An important issue for future research on pattern learning will be to examine the similarities and differences among pattern learning in the spatial, temporal, and magnitude domains. Given the pervasiveness of patterns, and their importance for responding to the causal structure of the world, it seems quite likely that common mechanisms have evolved for the detection of patterns across these stimulus dimensions.

\section{REFERENCES}

Brown, M. F., \& Moore, J. A. (1997). In the dark II: Spatial choice when access to extrinsic cues is eliminated. Animal Learning \& Behavior, 25, 335-346.

Brown, M. F., \& Terrinoni, M. (1996). Control of choice by the spatial configuration of goals. Journal of Experimental Psychology: Animal Behavior Processes, 22, 438-446.

Cartwright, B. A., \& Collette, T. S. (1983). Landmark learning in bees: Experiments and models. Journal of Comparative Physiology, 151, 521-543.

Cheng, K. (1986). A purely geometric module in the rat's spatial representation. Cognition, 23, 149-178.

Cheng, K. (1989). The vector sum model of pigeon landmark use. Journal of Experimental Psychology: Animal Behavior Processes, 15, 366-375.

ChEng, K., \& Gallistel, C. R. (1984). Testing the geometric power of an animal's spatial representation. In H. L. Roitblat, T. G. Bever, \& H. S. Terrace (Eds.), Animal cognition (pp. 409-423). Hillsdale, NJ: Erlbaum.

Cheng, K., \& SPETCH, M. L. (1999). Landmark-based spatial memory in pigeons. In R. G. Cook (Ed.), Avian visual cognition [On-line]. Available: www.pigeon.psy.tufts.edu/ave/

Church, R. M., \& LACOuRSE, D. M. (1998). Serial pattern learning of temporal intervals. Animal Learning \& Behavior, 26, 272-289.

Etienne, A. S., Maurer, R., \& Saucy, F. (1988). Limitations in the assessment of path dependent information. Behaviour, 106, 81-111.

ETIENNe, A. S., MAURER, R., \& SÉGuinot, V. (1996). Path integration in mammals and its interaction with visual landmarks. Journal of Experimental Biology, 199, 201-209.

Fountain, S. B., \& HulSE, S. H. (1981). Extrapolation of serial stimulus patterns by rats. Animal Learning \& Behavior, 9, 381-384.

GALLISTEL, C. R. (1990). The organization of learning. Cambridge, MA: MIT Press.

GreENE, C. M., \& Cook, R. G. (1997). Landmark geometry and identity controls spatial navigation in rats. Animal Learning \& Behavior, 25, 312-323.

HuLSE, S. H., \& DoRSKy, N. P. (1977). Structural complexity as a determinant of serial pattern learning. Learning \& Motivation, 8, 488-506.

Kamil, A. C., \& JoneS, J. E. (1997). The seed-storing corvid Clark's 
nutcracker learns geometric relationships among landmarks. Nature, 390, 276-279.

Lebowitz, B. K., \& Brown, M. F. (1999). Sex differences in spatial search and pattern learning in the rat. Psychobiology, 7, 364-371.

McNaughton, B. L., Chen, L. L., \& Markus, E. J. (1991). "Dead reckoning," landmark learning, and the sense of direction: A neurophysiological and computational hypothesis. Journal of Cognitive Neuroscience, 3, 190-202.

Morris, R. G. M. (1981). Spatial localization does not require the presence of local cues. Learning \& Motivation, 12, 239-260.

Olton, D. S. (1978). Characteristics of spatial memory. In S. H. Hulse, H. Fowler, \& W. K. Honig (Eds.), Cognitive processes in animal behavior (pp. 341-373). Hillsdale, NJ: Erlbaum.

Olton, D. S., \& Samuelson, R. J. (1976). Remembrance of places past: Spatial memory in rats. Journal of Experimental Psychology: Animal Behavior Processes, 2, 97-116.
PouCET, B. (1993). Spatial cognitive maps in animals: New hypotheses on their structure and neural mechanisms. Psychological Review, 100, 163-182.

Spetch, M. L., Cheng, K., MacDonald, S. E., Linkenhoker, B. A. Kelly, D. M., \& DoERKSON, B. A. (1997). Use of landmark configuration in pigeons and humans: Generality across search tasks. Journal of Comparative Psychology, 111, 14-24.

Thinus-Blanc, C. (1996). Animal spatial cognition: Behavioural and brain approaches. Singapore: World Scientific.

ZeILER, C. A., \& Brown, M. F. (2000). Spatial pattern learning in rats: Control by an iterative pattern. Manuscript in preparation.

(Manuscript received August 18, 1999; revision accepted for publication May 5, 2000.) 